\title{
I 8 SNPs of folate-related genes and risks of spina bifida and conotruncal heart defects
}

\author{
Gary M Shaw*1,4,5, Wei Lu², Huiping Zhu ${ }^{2}$, Wei Yang ${ }^{3}$, Farren BS Briggs ${ }^{4}$, \\ Suzan L Carmichael ${ }^{3,5}$, Lisa F Barcellos ${ }^{4}$, Edward J Lammer ${ }^{5}$ and \\ Richard H Finnell ${ }^{2}$
}

\begin{abstract}
Address: ${ }^{1}$ Department of Pediatrics, Division of Neonatal \& Developmental Medicine, Stanford University School of Medicine, Stanford, CA, USA, 2Institute of Biosciences and Technology, Texas A\&M Health Science Center, Houston, TX, USA, ${ }^{3}$ California Research Division, March of Dimes, California Research Division, Oakland, CA, USA, ${ }^{4}$ School of Public Health, University of California, Berkeley, School of Public Health, Berkeley, CA, USA and ${ }^{5}$ Children's Hospital Oakland Research Institute, Oakland, CA, USA

Email: Gary M Shaw* - gmshaw@stanford.edu; Wei Lu -wlu@ibt.tamhsc.edu; Huiping Zhu - hzhu@ibt.tamsch.edu; Wei Yang -WYang@marchofdimes.com; Farren BS Briggs - fbriggs@genepi.berkeley.edu; Suzan L Carmichael - SCarmichael@marchofdimes.com; Lisa F Barcellos - barcello@genepi.berkeley.edu; Edward J Lammer - elammer@chori.org; Richard H Finnell -rfinnell@ibt.tamhsc.edu

* Corresponding author
\end{abstract}

Published: 3 June 2009

BMC Medical Genetics 2009, 10:49 doi:10.1186/1471-2350-10-49
Received: 22 October 2008

Accepted: 3 June 2009

This article is available from: http://www.biomedcentral.com//47/-2350//0/49

(c) 2009 Shaw et al; licensee BioMed Central Ltd.

This is an Open Access article distributed under the terms of the Creative Commons Attribution License (http://creativecommons.org/licenses/by/2.0), which permits unrestricted use, distribution, and reproduction in any medium, provided the original work is properly cited.

\begin{abstract}
Background: Folic acid taken in early pregnancy reduces risks for delivering offspring with several congenital anomalies. The mechanism by which folic acid reduces risk is unknown. Investigations into genetic variation that influences transport and metabolism of folate will help fill this data gap. We focused on II 8 SNPs involved in folate transport and metabolism.

Methods: Using data from a California population-based registry, we investigated whether risks of spina bifida or conotruncal heart defects were influenced by 118 single nucleotide polymorphisms (SNPs) associated with the complex folate pathway. This case-control study included 259 infants with spina bifida and a random sample of 359 nonmalformed control infants born during 1983-86 or 1994-95. It also included 214 infants with conotruncal heart defects born during 1983-86. Infant genotyping was performed blinded to case or control status using a designed SNPlex assay. We examined single SNP effects for each of the II 8 SNPs, as well as haplotypes, for each of the two outcomes.

Results: Few odds ratios (ORs) revealed sizable departures from I.0. With respect to spina bifida, we observed ORs with $95 \%$ confidence intervals that did not include 1.0 for the following SNPs (heterozygous or homozygous) relative to the reference genotype: $B H M T$ ( $r s 3733890)$ OR = I.8 (I.I-3.I), CBS (rs285I39I) OR = 2.0 (I.2-3.1); CBS (rs2347I3) OR = 2.9 (I.3-6.7); MTHFDI (rs2236224) OR = I.7 (I.I-2.7); MTHFDI (hcvll 462908) OR = $0.2(0-0.9) ;$ MTHFD2 (rs702465) OR $=0.6(0.4-0.9) ;$ MTHFD2 (rs757। 842) OR = 0.6 (0.4-0.9); MTHFR ( $\mathrm{rs}$ I80I I33) OR = 2.0 (I.2-3.I); MTRR (rs I62036) OR = 3.0 (I.5-5.9); MTRR

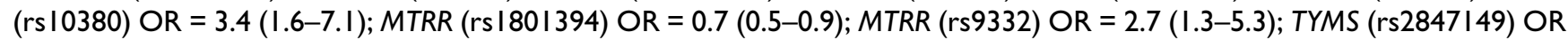
$=2.2$ (I.4-3.5); TYMS (rsI00I76I) OR = 2.4 (I.5-3.8); and TYMS (rs502396) OR = 2.I (I.3-3.3). However, multiple SNPs observed for a given gene showed evidence of linkage disequilibrium indicating that the observed SNPs were not individually contributing to risk. We did not observe any ORs with confidence intervals that did not include 1.0 for any of the studied SNPs with conotruncal heart defects. Haplotype reconstruction showed statistical evidence of nonrandom associations with TYMS, MTHFR, BHMT and MTR for spina bifida.
\end{abstract}

Conclusion: Our observations do not implicate a particular folate transport or metabolism gene to be strongly associated with risks for spina bifida or conotruncal defects. 


\section{Background}

Periconceptional vitamin supplementation with folic acid substantially reduces risks of women having neural tube defect-affected pregnancies [1,2] and has been implicated in reducing risks of several other congenital anomalies, including orofacial clefts and selected heart defects [3-11]. Mechanisms underlying these reduced risks have not been elucidated, although it has been speculated that supplementation with vitamins containing folic acid restores some normal developmental function that is genetically compromised in selected infants.

Investigating genetic variation that influences cellular absorption, transport, and metabolism of folate may offer insight into this unknown developmentally protective mechanism. Indeed, numerous investigations of genes that are specifically involved with folate metabolism have yielded at least one gene, 5, 10-methylenetetrahydrofolate reductase (MTHFR), that has been associated with a modest increased risk of neural tube defects (e.g., [12-17]), and possibly heart defects $[18,19]$. Observed risks with the two principal MTHFR variants, however, do not appear to account for a large proportion of the etiologic fraction of any of these defects, under the assumption that MTHFR variants have a causal role [17]. Thus, further investigation of other folate-related genes is necessary to reveal clues about mechanisms underlying the potential embryonic protective effects of folic acid supplementation.

We hypothesized that genetic susceptibility of fetal metabolism or transport of folate puts fetuses at risk for selected congenital anomalies. Using population-based data, we investigated 118 single nucleotide polymorphisms (SNPs) in 14 genes in the complex folate pathway as risk factors for spina bifida and conotruncal heart defects.

\section{Methods}

This population-based case-control study included infants with spina bifida or conotruncal heart defects diagnosed within 1 year after birth among infants and fetal deaths delivered to women residing in most California counties. Data were derived from the California Birth Defects Monitoring Program [20], a population-based active surveillance system for collecting information on infants and fetuses with congenital malformations. Diagnostic and demographic information was collected by program staff from multiple sources of medical records for all liveborn and stillborn fetuses (defined as >20 weeks gestation). Overall ascertainment for major malformations has been estimated as $97 \%$ complete [21]. Eligible were live born infants only because the source of DNA was from newborn screening cards.

Included were 259 infants with spina bifida and a random sample of 359 nonmalformed control infants born during
1983-86 and 1994-95 in selected counties in California. Also included for study were 214 infants with conotruncal heart defects, specifically d-transposition of the great arteries and tetralogy of Fallot. The random sample of 1983-86 controls for conotruncal heart defects included 220 of the overall 359. Newborn bloodspots were obtained from the State of California and their use in this study was consistent with the consent procedures at the time of sample collection. The protocol for this study was reviewed and approved by the State of California Health and Welfare Agency Committee for the Protection of Human Subjects.

Genomic DNA was extracted from dried blood spots on filter paper using the Puregene DNA Extraction Kit (Gentra, Minneapolis, MN). Prior to genotyping, genomic DNA was amplified using a commercial multiple displacement amplification (MDA) kit, GenomePhi (GE Healthcare, Piscataway, NJ). The MDA method relies on isothermal amplification using the DNA polymerase of the bacteriophage phi29 and is a recently developed technique for high performance WGA. MDA has been demonstrated to be reliable for genotyping, with the most favorable call rates, best genomic coverage, and lowest amplification bias [22]. Studies indicate no discernable difference between WGA samples with GenomiPhi kit and the original DNA templates [23,24]. The whole genome amplification (WGA) product was then quantified using RNase $P$ method (AppliedBiosystems, Foster City, CA). 150 ng WGA product was then used for each SNPlex assay pool which contained about 48 SNPs.

Genotype analyses were performed using SNPlex assays (AppliedBiosystems, Foster City, CA). SNP markers were selected using the SNPBrowser ${ }^{\mathrm{TM}}$ program (version 3.0) provided by AppliedBiosystems Inc. This program allowed selection of SNP markers from the HapMap database. For each target gene, tagging SNPs were selected based on the pairwise $r^{2}>=0.8$. SNPs with minor allele frequencies lower than $10 \%$ in Caucasians were excluded. All validated non-synonymous SNPs were included. Successful rates for SNPlex assays were $>96 \%$ for 75 SNPs, from $90 \%$ to $96 \%$ for 32 SNPs, from $70 \%$ to $90 \%$ for 7 SNPs. 15 SNPs suffered from more than $30 \%$ failure rates. In a subsequent effort to fill in the missing genotyping data and obtain higher call rate, we performed TaqMan SNP assays (Appliedbiosystems, Foster City, CA) for 22 of these SNPs on an ABI 7900 Genetic Analyzer.

All genotyping was performed blinded to subject's case or control status. Case and control infants were genotyped for 129 SNPs. Failure to obtain unambiguous genotype data on $>50 \%$ of the samples for 11 SNPs (CBS rs 1801181 and rs12329790; MTHFR rs1537514 and rs7533315; MTR rs10925257, NOS3 rs1800780 and hcv11631000; 
RFC1 rs1051266, rs4819130, hcv16186310, and rs7278825) resulted in their elimination from further analyses. The remaining 118 SNPs are shown in Table 1. The percentage of control study subjects (percentages were similar for cases) for whom genotype could be assigned is also shown in Table 1.

Genotypes among controls were analyzed to verify that their distributions fit Hardy-Weinberg expectations. Genotypes for each SNP were statistically consistent with Hardy-Weinberg expectations. Odds ratios and 95\% confidence intervals (CI) were used to estimate risks. These measures were calculated using SAS software (version 9.1). Information on maternal race/ethnicity was obtained for case and control infants from California birth certificates. Logistic regression was used to compute risk estimates adjusted for maternal race/ethnicity (white Hispanic; white nonHispanic, and other). Analyses estimated defect risks (spina bifida or conotruncal heart defects) for each SNP assuming a recessive model, i.e., homozygous variant genotype compared to homozygous reference genotype and heterozygous variant genotype compared to homozygous reference genotype. In addition to single SNP-at-a-time analyses, we explored haplotype block analyses. Haplotype analyses were performed using Haploview version 3.32. Identified blocks were assessed with odds ratios.

\section{Results}

Numbers of case and control infants stratified by race/ethnicity are shown in Table 2 . These data show the expected greater frequency of Hispanics in the spina bifida case group.

We examined risks for each of the 118 SNPs and for each of the two birth defect outcome (Additional file 1). Few odds ratios (ORs) revealed sizable departures from 1.0. Given the large number of comparisons $(n=472)$ we expected more ORs to be substantially different from 1.0 by chance. With respect to spina bifida, we observed ORs with confidence intervals that did not include 1.0 for the following SNPs (heterozygous or homozygous) relative to the reference genotype: $B H M T$ (rs3733890) $\mathrm{OR}=1.8$ (1.1-3.1), CBS (rs2851391) OR = 2.0 (1.2-3.1); CBS $(\mathrm{rs} 234713) \mathrm{OR}=2.9(1.3-6.7) ;$ MTHFD1 (rs2236224) $\mathrm{OR}=1.7(1.1-2.7) ;$ MTHFD1 $(\mathrm{hcv} 11462908) \mathrm{OR}=0.2$ (0-0.9); MTHFD2 (rs702465) OR = 0.6 (0.4-0.9); MTHFD2 (rs7571842) OR = 0.6 (0.4-0.9); MTHFR $(\operatorname{rs} 1801133) \mathrm{OR}=2.0(1.2-3.1) ; \operatorname{MTRR}(\mathrm{rs} 162036) \mathrm{OR}=$ 3.0 (1.5-5.9); MTRR (rs10380) OR = $3.4(1.6-7.1) ; M T R R$ $($ rs1801394) $\mathrm{OR}=0.7(0.5-0.9) ; \operatorname{MTRR}(\mathrm{rs} 9332) \mathrm{OR}=2.7$ (1.3-5.3); TYMS (rs2847149) OR = $2.2(1.4-3.5) ; T Y M S$ $($ rs1001761) OR = $2.4(1.5-3.8) ;$ and TYMS (rs502396) $\mathrm{OR}=2.1$ (1.3-3.3). Each gene involving multiple SNP associations was investigated for linkage disequilibrium.
Modest to strong evidence for linkage disequilibrium was observed for SNPs in each gene, i.e., D' ranged from 0.44 to 1.0 with all $\mathrm{p}$ values $<10^{-4}$. With respect to conotruncal heart defects, we did not observe any OR with a confidence interval that did not include 1.0.

We did not observe evidence to indicate that risk patterns were confounded by race/ethnicity groupings, i.e., observed ORs were not substantially altered after adjusting for maternal race/ethnicity (not shown, available from authors upon request).

Haplotypes, reconstructed for each gene based on studied SNPs, were explored to assess risks for each case group. A total of 77 of the 118 studied SNPs formed 17 haplotype blocks. As shown in Table 3, blocks for TYMS, MTHFR, $B H M T$, and MTR showed some evidence of nonrandom effects for spina bifida. For each of these haplotypes we observed decreased risk associated with the lower frequency haplotype relative to the most frequent haplotype. Similar to SNP analyses, haplotype analyses for conotruncal heart defects did not reveal evidence of nonrandom effects, with the exception of one haplotype block for MTR (Table 4).

Haplotype analyses were stratified by race/ethnic background (Hispanic white and nonHispanic white). We observed evidence of a nonrandom haplotype association with TYMS for spina bifida and conotruncal heart defects among nonHispanic whites. Lack of evidence for other haplotypes that were observed overall was likely the result of smaller sample sizes from stratification.

\section{Discussion}

In this California population we found only modest evidence that polymorphisms in 14 folate-related genes contributed to risk of spina bifida. SNPs contributing risks were in BHMT, CBS, MTHFD1, MTHFD2, MTHFR, MTRR, and TYMS. Haplotype association analyses further identified TYMS and MTHFR as potential contributors to spina bifida risk. In general, however, most of these folaterelated genes showed little evidence for a gene-only effect on risk of spina bifida, and even less, on risks of conotruncal heart defects.

The 14 genes studied here have been implicated in the complex metabolic cycle involving folate (e.g., [25-27]). To our knowledge, this study contained the largest number of SNPs in folate-related genes interrogated as risk factors for human spina bifida or conotruncal heart defects. Previous studies have included some of the SNPs examined here. For example, Boyles and colleagues [28] studied 28 SNPs in 11 folate-related genes and found that only BHMT (rs3733890) was associated with increased 
Table I: Fourteen folate-related genes and I I8 SNPs

\begin{tabular}{|c|c|c|c|c|c|c|}
\hline Gene & Change & Chromosome & Base Position & SNP_ID & Type/Comment & Percent Genotyped' \\
\hline BHMT & $R(A / G)$ & 5 & 78457715 & rs3733890 & exon, nonsynonymous R239Q & 100 \\
\hline BHMT & $Y(C / T)$ & 5 & 78471967 & rs1915706 & Intergenic/Unknown & 96.4 \\
\hline BHMT & $(\mathrm{G} / \mathrm{C})$ & 5 & 78567093 & rs|316753 & Tag, BHMT & 100 \\
\hline BHMT & $M(C / A)$ & 5 & 78465350 & rs617219 & intergenic & 96.4 \\
\hline BHMT & $M(A / C)$ & 5 & 78438303 & rs645II2 & Intergenic/Unknown & 96.9 \\
\hline BHMT & $\mathrm{W}(\mathrm{A} / \mathrm{T})$ & 5 & 78462964 & rs585800 & untranslated region & 94.2 \\
\hline BHMT & $S(C / G)$ & 5 & 78559288 & rs3829809 & Tag, BHMT & 100 \\
\hline BHMT & $\mathrm{Y}(\mathrm{C} / \mathrm{T})$ & 5 & 78452172 & rs567754 & intron & 95.8 \\
\hline BHMT2 & $M(A / C)$ & 5 & 78400443 & rs642431 & intergenic-BHMT2;intron-DMGDH & 91.1 \\
\hline BHMT2 & $R(A / G)$ & 5 & 78405657 & rs626105 & intron & 96.1 \\
\hline BHMT2 & $\mathrm{Y}(\mathrm{C} / \mathrm{T})$ & 5 & 78409187 & rs682985 & exon, synonymous & 95.5 \\
\hline BHMT2 & $M(A / C)$ & 5 & 78387392 & rs2253262 & exon, synonymous & 96.4 \\
\hline BHMT2 & $\mathrm{K}(\mathrm{G} / \mathrm{T})$ & 5 & 78402082 & rs670220 & Validated & 96.7 \\
\hline BHMT2 & $R(A / G)$ & 5 & 78404048 & rs592052 & intron & 99.2 \\
\hline BHMT2 & $R(A / G)$ & 5 & 78419219 & rs597560 & intron & 98.3 \\
\hline CBS & $\mathrm{Y}(\mathrm{T} / \mathrm{C})$ & 21 & 43360473 & rs285|39| & intron & 92.5 \\
\hline CBS & $\mathrm{R}(\mathrm{A} / \mathrm{G})$ & 21 & 43359173 & rs2298759 & intron & 72.4 \\
\hline CBS & $Y(T / C)$ & 21 & 43361102 & rs2347|4 & intron & 90 \\
\hline CBS & $S(C / G)$ & 21 & 43346936 & rs1051319 & untranslated region & 91.9 \\
\hline CBS & $Y(T / C)$ & 21 & 43376503 & rs234784 & Tag, CBS & 99.7 \\
\hline CBS & $\mathrm{N}(\mathrm{A} / \mathrm{C} / \mathrm{G} / \mathrm{T})$ & 21 & 43346760 & $r s 12613$ & untranslated region & 92.5 \\
\hline CBS & $S(C / G)$ & 21 & 43377074 & $r s 234785$ & Tag, CBS & 100 \\
\hline CBS & $R(A / G)$ & 21 & 43360960 & rs234713 & intron & 91.1 \\
\hline CBS & $\mathrm{Y}(\mathrm{C} / \mathrm{T})$ & 21 & 43376312 & rs234783 & Tag, CBS & 100 \\
\hline DHFR & $\mathrm{Y}(\mathrm{C} / \mathrm{T})$ & 5 & 79986537 & rs1650697 & Validated nsSNP & 92.2 \\
\hline DHFR & $\mathrm{W}(\mathrm{A} / \mathrm{T})$ & 5 & 79957572 & rs 12109877 & Validated & 94.2 \\
\hline DHFR & $\mathrm{Y}(\mathrm{C} / \mathrm{T})$ & 5 & 79987790 & rs380691 & Validated & 95.5 \\
\hline DHFR & $M(A / C)$ & 5 & 79985331 & rs|478834 & Validated & 96.4 \\
\hline DHFR & $Y(C / T)$ & 5 & 79966012 & rsl643638 & Validated & 92.8 \\
\hline DHFR & $M(A / C)$ & 5 & 79961366 & rs 2618372 & Validated & 96.9 \\
\hline DHFR & $R(A / G)$ & 5 & 79980489 & $r s|3| 6 \mid 245$ & Validated & 96.1 \\
\hline DHFR & $Y(C / T)$ & 5 & 79975899 & rs 1643650 & Validated & 94.7 \\
\hline DHFR & $\mathrm{K}(\mathrm{G} / \mathrm{T})$ & 5 & $7998 \mid 467$ & rs83682I & Validated & 97.5 \\
\hline FOLRI & $\mathrm{Y}(\mathrm{C} / \mathrm{T})$ & 11 & 73373406 & rs 1540087 & untranslated region & 95.8 \\
\hline FOLRI & $\mathrm{W}(\mathrm{T} / \mathrm{A})$ & 11 & 73380857 & rsII 235462 & Tag, FOLRI & 100 \\
\hline FOLRI & $R(A / G)$ & 11 & 73372879 & rs2071010 & untranslated region & 91.9 \\
\hline FOLR2 & $R(A / G)$ & 11 & 73404256 & rs2298444 & intron & 92.2 \\
\hline FOLR2 & $R(A / G)$ & 11 & 73402049 & rs5।4933 & intron & 100 \\
\hline FOLR2 & $\mathrm{W}(\mathrm{A} / \mathrm{T})$ & 11 & 73401368 & rs651646 & untranslated region & 100 \\
\hline MTHFDI & $Y(C / T)$ & 14 & 63984935 & rs2236222 & intron & 95.5 \\
\hline MTHFDI & $Y(C / T)$ & 14 & 63978904 & rs2236224 & intron & 97.8 \\
\hline MTHFDI & $\mathrm{Y}(\mathrm{C} / \mathrm{T})$ & 14 & 63952133 & rs1950902 & exon, nonsynonymous & 90.5 \\
\hline MTHFDI & $Y(C / T)$ & 14 & 63978598 & rs2236225 & exon, nonsynonymous GI958A (R653Q) & 100 \\
\hline MTHFDI & $(\mathrm{T} / \mathrm{A})$ & 14 & 63999040 & hCVII 462908 & Tag, MTHFDI & 100 \\
\hline MTHFDI & $R(A / G)$ & 14 & 63957808 & hCVII 660794 & intron & 95.3 \\
\hline MTHFDI & $R(A / G)$ & 14 & 63988165 & rsII 849530 & intron & 95.8 \\
\hline MTHFDI & $R(A / G)$ & 14 & 63990418 & rs $1256 \mid 46$ & intron & 95 \\
\hline MTHFD I & $\mathrm{Y}(\mathrm{C} / \mathrm{T})$ & 14 & 63985918 & rs 10137921 & exon, nonsynonymous & 96.4 \\
\hline MTHFD I & $Y(C / T)$ & 14 & 63980547 & rs $1256 \mid 42$ & intron & 97.8 \\
\hline MTHFD2 & $\mathrm{Y}(\mathrm{T} / \mathrm{C})$ & 2 & 74304595 & rsllII26426 & Intergenic, Tag & 100 \\
\hline MTHFD2 & $(\mathrm{T} / \mathrm{A})$ & 2 & 74280806 & rs702465 & Intergenic, Tag & 96.7 \\
\hline MTHFD2 & $R(A / G)$ & 2 & 74313429 & rs1667599 & Intergenic, Tag & 100 \\
\hline MTHFD2 & $R(A / G)$ & 2 & 74340847 & rsl667627 & Validated & 96.1 \\
\hline MTHFD2 & $\mathrm{W}(\mathrm{A} / \mathrm{T})$ & 2 & 74333849 & rs828858 & Intergenic, Tag & 100 \\
\hline MTHFD2 & $(\mathrm{C} / \mathrm{G})$ & 2 & 74281605 & rs702466 & Intergenic, Tag & 99.7 \\
\hline MTHFD2 & $\mathrm{R}(\mathrm{A} / \mathrm{G})$ & 2 & 74372559 & rs757I842 & Intergenic, Tag & 100 \\
\hline MTHFD2 & $R(A / G)$ & 2 & 74348376 & rs828903 & Validated & 94.4 \\
\hline MTHFR & $R(A / G)$ & I & 11801310 & rs3737964 & Validated & 95.8 \\
\hline MTHFR & $R(A / G)$ & i & 11823734 & rs535107 & Intergenic, Tag & 93.3 \\
\hline MTHFR & $\mathrm{K}(\mathrm{G} / \mathrm{T})$ & 1 & I I 798240 & rs1931226 & Validated & 96.9 \\
\hline
\end{tabular}


Table I: Fourteen folate-related genes and II 8 SNPs (Continued)

\begin{tabular}{|c|c|c|c|c|c|c|}
\hline MTHFR & $\mathrm{R}(\mathrm{A} / \mathrm{G})$ & $T$ & 11780518 & rs4846048 & Validated & 89.7 \\
\hline MTHFR & $\mathrm{Y}(\mathrm{C} / \mathrm{T})$ & I & 11796598 & rs7525338 & Validated & 97.5 \\
\hline MTHFR & $R(A / G)$ & I & 11785193 & rs2274976 & exon, nonsynonymous & 93 \\
\hline MTHFR & $Y(C / T)$ & 1 & 11792217 & rs4846052 & intron & 96.9 \\
\hline MTHFR & $\mathrm{Y}(\mathrm{C} / \mathrm{T})$ & 1 & 11790644 & rs 1801133 & exon, nonsynonymous C677T & 99.4 \\
\hline MTHFR & $R(A / G)$ & 1 & 11775209 & rsl889292 & Intergenic, Tag & 100 \\
\hline MTHFR & $Y(C / T)$ & 1 & 11797323 & rs2066470 & exon, synonymous & 95.3 \\
\hline MTHFR & $R(A / G)$ & 1 & I I 788723 & rs484605I & exon, synonymous & 93 \\
\hline MTHFR & $R(A / G)$ & 1 & 11786566 & rs|4764I3 & intron & 93.9 \\
\hline MTHFR & $M(A / C)$ & I & I I 788742 & rs $|80| 13 \mid$ & exon, nonsynonymous AI298C & 99.7 \\
\hline MTR & $M(A / C)$ & 1 & 233374717 & rs2275565 & Validated & 96.1 \\
\hline MTR & $Y(C / T)$ & 1 & 233322616 & rs|806505 & intron & 97.5 \\
\hline MTR & $K(G / T)$ & 1 & 233386474 & rs382057I & Validated & 96.1 \\
\hline MTR & $\mathrm{Y}(\mathrm{C} / \mathrm{T})$ & 1 & 233335898 & rs3754255 & Validated & 94.7 \\
\hline MTR & $S(C / G)$ & 1 & 233381346 & rs 10802569 & Validated & 96.1 \\
\hline MTR & $R(A / G)$ & 1 & 233376992 & rs 1266164 & intron & 96.1 \\
\hline MTR & $R(A / G)$ & I & $23337454 \mid$ & rs|805087 & exon, nonsynonymous A2756G & 96.4 \\
\hline MTR & $\mathrm{W}(\mathrm{A} / \mathrm{T})$ & I & 233385428 & rs4659743 & Validated & 98.3 \\
\hline MTR & $\mathrm{K}(\mathrm{G} / \mathrm{T})$ & 1 & 233390667 & rs6676866 & Validated & 98.3 \\
\hline MTR & $S(C / G)$ & I & 233315110 & rs 12060570 & Validated & 98.6 \\
\hline MTR & $\mathrm{W}(\mathrm{A} / \mathrm{T})$ & 1 & 233306545 & rs 955516 & Validated & 99.2 \\
\hline MTR & $\mathrm{K}(\mathrm{G} / \mathrm{T})$ & 1 & $2333|383|$ & rs4077829 & intron & 96.7 \\
\hline MTR & $R(A / G)$ & 1 & 233364202 & rsl770449 & intron & 94.4 \\
\hline MTR & $S(C / G)$ & 1 & 233353709 & rs3768I39 & intron & 95.5 \\
\hline MTR & $R(A / G)$ & I & 233300165 & rs4659724 & intron & 97.2 \\
\hline MTR & $Y(C / T)$ & 1 & 233327367 & rs6668344 & intron & 96.4 \\
\hline MTR & $R(A / G)$ & 1 & 233367345 & rs7367859 & Validated & 93.9 \\
\hline MTR & $K(G / T)$ & 1 & 233354605 & rs3768|42 & Validated & 96.1 \\
\hline MTR & $Y(C / T)$ & I & 233348403 & rs 10925252 & Validated & 96.9 \\
\hline MTR & $R(A / G)$ & I & 233380610 & rs2229276 & exon, synonymous & 95 \\
\hline MTR & $R(A / G)$ & 1 & 233388346 & rsl050993 & untranslated region & 97.2 \\
\hline MTRR & $R(A / G)$ & 5 & 7938959 & rsl62036 & Validated nsSNP Lys/Arg & 95.5 \\
\hline MTRR & $S(C / G)$ & 5 & 7944506 & rsI6879334 & exon, nonsynonymous Pro/Arg & 90 \\
\hline MTRR & $R(G / A)$ & 5 & 7950319 & rs 1802059 & exon, synonymous & 94.7 \\
\hline MTRR & $R(G / A)$ & 5 & 7942216 & rs2287779 & exon, synonymous & 97.2 \\
\hline MTRR & $R(A / G)$ & 5 & 7927847 & rs326120 & intron & 87.7 \\
\hline MTRR & $Y(C / T)$ & 5 & 7950191 & rsl0380 & exon, nonsynonymous, His/Tyr & 96.4 \\
\hline MTRR & $R(A / G)$ & 5 & 7923973 & rs1801394 & exon, nonsynonymous & 96.7 \\
\hline MTRR & $Y(C / T)$ & 5 & 7953712 & rs9332 & UTR & 92.2 \\
\hline MTRR & $S(C / G)$ & 5 & 7938907 & rs 10064631 & exon, nonsynonymous & 95 \\
\hline MTRR & $\mathrm{W}(\mathrm{A} / \mathrm{T})$ & 5 & 7931424 & rs 2303080 & exon, nonsynonymous & 96.1 \\
\hline MTRR & $\mathrm{R}(\mathrm{A} / \mathrm{G})$ & 5 & 7949511 & rs3776455 & intron & 95 \\
\hline MTRR & $R(A / G)$ & 5 & 7931179 & rsl532268 & exon, nonsynonymous & 95 \\
\hline MTRR & $R(A / G)$ & 5 & 7945310 & rsl62048 & intron & 98.6 \\
\hline NOS3 & $R(A / G)$ & 7 & $150 \mid 45737$ & rs891512 & intron & 86.6 \\
\hline NOS3 & $R(A / G)$ & 7 & $|50| 2759 \mid$ & rs 1800779 & untranslated region & 87.5 \\
\hline NOS3 & $\mathrm{Y}(\mathrm{C} / \mathrm{T})$ & 7 & $150 \mid 48555$ & rs391821I & exon, synonymous & 96.9 \\
\hline RFCl & $\mathrm{K}(\mathrm{G} / \mathrm{T})$ & 21 & 45761011 & rs3788189 & intron & 81.1 \\
\hline RFCl & $R(A / G)$ & 21 & 45755537 & rs 12483377 & Tag, RFC & 100 \\
\hline RFCl & $R(A / G)$ & 21 & 45756112 & rs2236484 & Intron, Tag & 98.6 \\
\hline RFCl & $R(A / G)$ & 21 & 45761386 & rs3788190 & Intron, Tag & 91.1 \\
\hline RFCl & $S(C / G)$ & 21 & 45750430 & rs 10483080 & intron & 99.7 \\
\hline $\mathrm{RFCl}$ & $\mathrm{Y}(\mathrm{C} / \mathrm{T})$ & 21 & 45777720 & rs 2330183 & intron & 91.4 \\
\hline TYMS & $\mathrm{Y}(\mathrm{C} / \mathrm{T})$ & 18 & 652215 & rsII540I52 & exon, nonsynonymous & 95.8 \\
\hline TYMS & $Y(C / T)$ & 18 & 660414 & rs 2853532 & intron & 96.4 \\
\hline TYMS & $R(A / G)$ & 18 & 65637I & rs2847|49 & intron & 97.2 \\
\hline TYMS & $Y(C / T)$ & 18 & 652103 & rsl00176I & intron & 98.9 \\
\hline TYMS & $\mathrm{Y}(\mathrm{C} / \mathrm{T})$ & 18 & 649236 & rs502396 & intron & 97.8 \\
\hline
\end{tabular}

'Percent of 359 controls genotyped for each SNP.

Abbreviations: $B H M T$ = betaine homocysteine methyltransferase; $B H M T 2$ betaine homocysteine methyltransferase-2; $C B S=c y s t a t h i o n e$ beta synthase; DHFR = dihydrofolate reductase; FOLRI folate receptor I; FOLR2 folate receptor $2 ; M T H F D I=$ methylenetetrahydrofolate dehydrogenase I; $M T H F D 2=$ methylenetetrahydrofolate dehydrogenase $2 ; M T H F R=$ methylenetetrahydrofolate reductase; $M T R=$ methionine synthase; $M T R R=$ methionine synthase reductase; NOS3 = nitric oxide synthase; $R F C I=$ reduced folate carrier I; TYMS = thymidylate synthase. 
Table 2: Racial/ethnic percentages of malformed cases and nonmalformed controls, California 1983-86 and 1994-95.

\begin{tabular}{lcccc}
\hline & \multicolumn{2}{c}{ Spina Bifida } & \multicolumn{2}{c}{ Conotruncal Heart } \\
& $\begin{array}{c}\text { Cases } \\
\mathrm{n}=259 \\
\%^{2}\end{array}$ & $\begin{array}{c}\text { Controls } \\
\mathrm{n}=359 \\
\%^{2}\end{array}$ & $\begin{array}{c}\text { Cases } \\
\mathrm{n}=214 \\
\%^{2}\end{array}$ & $\begin{array}{c}\text { Controls } \\
\mathrm{n}=220^{1} \\
\%^{2}\end{array}$ \\
& & & & \\
Race/Ethnicity & & & & \\
$\begin{array}{l}\text { White, Hispanic } \\
\text { White, nonHispanic }\end{array}$ & 50.6 & 31.5 & 17.8 & 18.6 \\
Other & 12.0 & 47.4 & 53.3 & 61.8 \\
& & 20.6 & 26.2 & 18.6 \\
\hline
\end{tabular}

IThe number of controls born in the period 1983-86 among the 359 selected for the overall study period 1983-86 and 1994-95. The 220 represent the birth years of cases with conotruncal heart defects.

2 Percentages may not equal 100 owing to missing data or rounding.

spina bifida risk. This BHMT association is consistent with our findings that showed an odds ratio of $1.8(1.1-3.1)$.

Many studies have explored MTHFR 677 (rs1801133) polymorphism. A range of risks, including no-effect, has been reported for this SNP relative to spina bifida. Botto and Yang [15] in a meta-analysis demonstrated a pooled odds ratio of 1.8 for spina bifida among infants homozygous for 677T. A few studies have also explored this 677 SNP in MTHFR as a risk factor for selected congenital heart defects, with most investigations finding no or little association [18,19,29-31]. We did observe a 2fold increased risk of spina bifida associated with this SNP for homozygous infants. Further, haplotype analyses showed some association for the MTHFR gene as well.

Methionine synthase (MTR) is a vitamin $B_{12}$ dependent enzyme that is essential for the remethylation of homocysteine to methionine. The enzyme is required by cells for the essential accumulation of folate [32]. One particular SNP (A2756G; rs1805087) has been considerably investigated, with increased risks of NTDs reported in some studies [33-35], but not in others [36,37]. We did not find an increased risk for spina bifida or conotruncal heart defects associated with this SNP or any other SNP of MTR.

Cystathione beta synthase (CBS) is critical to the degradation of homocysteine to cysteine. Regulation of this pyridoxal phosphate-dependent enzyme catalyzes the hydroxyl group of serine with the thiolate of homocysteine [38]. The polymorphism in the CBS gene that has received the most study is a 68 bp insertion (844ins68), with predominantly no associations observed for NTDs [27]. This polymorphism was not investigated in the current study. We did observe, however, two CBS SNPs (rs2851391 and rs234713) that showed increased risks for spina bifida. Boyles et al [28], albeit using a different study design than ours, observed that these two SNPs were not differentially transmitted from parents of infants with spina bifida.

MTRR gene polymorphisms (particularly rs1801394) have been investigated as a risk factor for both spina bifida and congenital heart defects. Polymorphisms in MTRR could alter homocysteine levels because methionine synthase reductase participates in maintaining the vitamin $\mathrm{B}_{12}$-dependent conversion of homocysteine to methionine [32]. The most frequently studied MTRR polymorphism has been the 66A>G (rs1801394). This polymorphism in infants was associated with a 2.6-fold increased risk of spina bifida in an earlier study by us [33], it was associated with increased risk for spina bifida in another study only when vitamin $B_{12}$ levels were low [39], or in combination with MTHFR CC genotype [35]. The polymorphism in mothers of infants with neural tube defects has been associated with increased risk in one study [40], but not in another study [41]. Recent work from the Netherlands has shown a lack of association between this polymorphism and risk for conotruncal heart defects [42] as well as no increased risks for a broader phenotypic group of heart defects [43]. In this study, the $66 \mathrm{~A}>\mathrm{G}$ polymorphism was not associated with increased risks for either spina bifida or conotruncal heart defects. We did observe, however, approximately 3-fold elevated risks for spina bifida associated with three other MTRR SNPs (rs162036, rs10380, and rs9332). The significance of these observations will have to be explored in future studies.

With respect to MTHFD1 and MTHFD2, two studies have demonstrated an association with one polymorphism (rs 2236225) in MTHFD1 and NTD risk. One study showed a 1.5-fold increase in risk of an NTD-affected pregnancy in Irish women who were homozygous AA [44], a finding that confirmed an earlier increased risk that was identified in Irish women. Another study showed a similar risk for Italian women as well as a 1.9-fold risk for infants with the AA genotype to have spina bifida [45]. For this particular SNP, we observed a similar magnitude of risk $(\mathrm{OR}=1.6)$ for infants with the homozygous genotype, but the estimate was relatively imprecise. We did observe a modestly elevated spina bifida risk for individuals who were homozygous for another MTHFD1 SNP (rs2236224) and modestly lowered risks for three others (hcv11462908, rs702465, and rs7571842). These observations will need to be replicated in future studies.

Polymorphisms in the DHFR gene have not been wellstudied for their role in risks of birth defects. Three studies have investigated a 19-bp deletion with mixed results [4648]. That particular polymorphism was not interrogated in the current study. 
Table 3: Haplotype associations with risks of spina bifida

\begin{tabular}{|c|c|c|}
\hline Haplotype Block & Frequency & Odds Ratio (95\% Cl) \\
\hline \multicolumn{3}{|l|}{ TYMS } \\
\hline CGC & 0.500 & REF \\
\hline TAT & 0.373 & $0.7(0.6-0.9)$ \\
\hline TAC & 0.115 & $0.5(0.3-0.7)$ \\
\hline \multicolumn{3}{|l|}{ MTRR } \\
\hline ATTAGCAACAC & 0.264 & REF \\
\hline ACTGGCAGTGT & 0.213 & $1.4(1.0-1.9)$ \\
\hline ACTAGCAACGC & 0.201 & $0.8(0.6-1.1)$ \\
\hline GCTAGCGGCGC & 0.162 & I.I (0.7-I.5) \\
\hline ACAAAGAGCGC & 0.055 & I.I (0.7-I.9) \\
\hline ACTAGCAGCGC & 0.034 & $0.6(0.3-1.3)$ \\
\hline ACTAAGAGCGC & 0.027 & $1.2(0.6-2.6)$ \\
\hline ACTGGCAGCGT & 0.011 & $1.4(0.5-4.1)$ \\
\hline \multicolumn{3}{|l|}{ MTHFR* } \\
\hline GGG & 0.656 & REF \\
\hline AGA & 0.163 & $0.9(0.6-1.2)$ \\
\hline AGG & 0.121 & $0.9(0.6-1.2)$ \\
\hline AAA & 0.057 & $0.6(0.3-1.0)$ \\
\hline \multicolumn{3}{|l|}{ MTHFR** } \\
\hline TCCCA & 0.368 & REF \\
\hline CCCCA & 0.231 & $0.7(0.5-0.9)$ \\
\hline СTCTG & 0.180 & $0.8(0.6-1.1)$ \\
\hline CTTCG & 0.099 & $0.6(0.4-0.9)$ \\
\hline CTCCG & 0.063 & $0.7(0.5-1.2)$ \\
\hline CTCCA & 0.037 & $1.0(0.5-1.8)$ \\
\hline \multicolumn{3}{|l|}{ CBS } \\
\hline CG & 0.889 & REF \\
\hline TC & 0.055 & $1.2(0.7-1.9)$ \\
\hline $\mathrm{CC}$ & 0.053 & $0.6(0.3-1.0)$ \\
\hline \multicolumn{3}{|l|}{ RFCI* } \\
\hline CG & 0.856 & REF \\
\hline GG & 0.079 & I.I (0.7-I.7) \\
\hline GA & 0.063 & $1.0(0.6-1.6)$ \\
\hline \multicolumn{3}{|l|}{ RFCI ${ }^{* *}$} \\
\hline TG & 0.486 & REF \\
\hline GA & 0.463 & $0.9(0.7-1.2)$ \\
\hline GG & 0.046 & $0.6(0.3-1.0)$ \\
\hline \multicolumn{3}{|l|}{ MTHFDI* } \\
\hline CT & 0.486 & REF \\
\hline TC & 0.429 & I.3 (I.0-1.6) \\
\hline $\mathrm{CC}$ & 0.080 & $0.9(0.6-1.4)$ \\
\hline \multicolumn{3}{|l|}{ MTHFDI** } \\
\hline GT & 0.825 & REF \\
\hline AA & 0.167 & $0.9(0.6-1.2)$ \\
\hline \multicolumn{3}{|l|}{ FOLR2 } \\
\hline TA & 0.549 & REF \\
\hline AG & 0.356 & $1.0(0.8-1.3)$ \\
\hline $\mathrm{AA}$ & 0.093 & I.0 (0.7-I.6) \\
\hline \multicolumn{3}{|l|}{ MTHFD2* } \\
\hline TA & 0.589 & REF \\
\hline $\mathrm{CA}$ & 0.321 & I.I (0.9-I.4) \\
\hline CG & 0.089 & I.I (0.7-I.6) \\
\hline \multicolumn{3}{|l|}{ MTHFD2** } \\
\hline $\mathrm{TC}$ & 0.388 & REF \\
\hline TT & 0.332 & I.2(0.9-I.5) \\
\hline AT & 0.276 & $1.0(0.8-1.4)$ \\
\hline \multicolumn{3}{|l|}{ ВНMT2 } \\
\hline GGGTCA & 0.466 & REF \\
\hline TAACTC & 0.219 & $\mathrm{I} .0(0.7-\mathrm{I} .3)$ \\
\hline
\end{tabular}


Table 3: Haplotype associations with risks of spina bifida (Continued)

\begin{tabular}{|c|c|c|}
\hline GAGCTC & 0.171 & I.I (0.8-1.6) \\
\hline GAGTCA & 0.091 & $1.0(0.6-1.5)$ \\
\hline GGGTCC & 0.022 & $0.7(0.3-1.7)$ \\
\hline \multicolumn{3}{|l|}{ BHMT* } \\
\hline CAA & 0.339 & REF \\
\hline TGA & 0.326 & $0.7(0.5-0.9)$ \\
\hline CGT & 0.172 & $0.7(0.5-1.0)$ \\
\hline CGA & 0.158 & $0.9(0.6-1.2)$ \\
\hline \multicolumn{3}{|l|}{$B H M T^{* *}$} \\
\hline $\mathrm{AC}$ & 0.501 & REF \\
\hline CT & 0.373 & $0.8(0.7-1.1)$ \\
\hline AT & 0.120 & $0.9(0.6-1.3)$ \\
\hline \multicolumn{3}{|l|}{ DHFR } \\
\hline CTTACCA & 0.402 & REF \\
\hline CTTACCG & 0.390 & $0.9(0.7-1.2)$ \\
\hline ACCGAAA & 0.201 & $0.9(0.7-1.3)$ \\
\hline \multicolumn{3}{|l|}{ MTR } \\
\hline AATCTTTCCTAGAGGGCTTGG & 0.373 & REF \\
\hline GTGGCCCTGGGAAGAAGAGAT & 0.262 & $1.0(0.7-1.3)$ \\
\hline GTGGCCCTCTAGGTGACTTGG & 0.190 & $0.9(0.7-1.3)$ \\
\hline GTGGCCCTGGGGAGAAGAGAT & 0.045 & $1.4(0.8-2.5)$ \\
\hline GTGGCCTTCTAGATGACTTGT & 0.040 & $0.6(0.3-1.2)$ \\
\hline GTGGCCCTCGAAAGGAGTTGT & 0.032 & $0.3(0.1-0.6)$ \\
\hline
\end{tabular}

TYMS included rs 100176I, rs2847|49 and, rs2853532; MTRR included rs326120, rs I532268, rs2303080, rs |62036, rs2287779, rs I6879334, rs 162048 , rs 3776455, rs 10380

rs 1802059, and rs9332; MTHFR* included rs 1889292, rs2274976, and rs 14764 I3; MTHFR** included rs |80 I I33, rs4846052, rs2066470, rs3737964, and rs535I07; CBS included rs I26I3 and rs I05I319; RFCI* included rs 10483080 and rs I2483377; RFCI** included rs3788I89 and rs3788 I90; MTHFDI* included rs2236224 and rs 1256I42; MTHFDI** included rs I 256I46 and hcvI I462908; FOLR2 included rs65I646 and rs5 I4933; MTHFD2* included rs I I 26426 and rs 1667599; MTHFD2** included rs828858 and rs 1667627; BHMT2 included rs670220, rs592052, rs626105, rs682985, rs597560, and rs645। I2; BHMT* included rs567754, rs3733890, and rs585800; BHMT** included rs6I72 I9 and rs 1915706; DHFR included rs26I8372, rs 1643638, rs 1643650, rs I3I6I245, rs83682I, rs 1478834, and rs38069I; MTR included rs4659724, rs9555I6, rs4077829, rs 12060570, rs 1806505, rs6668344, rs3754255, rs 10925252, rs3768I39, rs3768|42, rs I770449, rs7367859, rs I805087, rs2275565, rs1266I64, rs2229276, rs 10802569, rs4659743, rs382057I, rs 1050993, and rs6676866.

Our analyses did not show associations with SNPs in RFC1. Previous investigations of this gene have focused on a particular SNP, rs1051266, and have found mixed results [37,41,49-53]. This particular SNP was not analyzed here as a result of too many samples failing to be genotyped for this SNP using the SNPlex platform.

Recent studies have focused on the importance of TYMS in the folate metabolic pathway, including associations between TYMS polymorphisms and folate levels [54-56].
This folate-dependent enzyme catalyzes the reductive methylation of deoxyuridylate (dUMP) to thymidylate (dTMP), thereby playing a central role in DNA synthesis and repair by serving as the primary intracellular source of dTMP [54,57-59]. We previously [56] observed a 4-fold increased risk of spina bifida in nonHispanic white infants who had a polymorphism for a $28 \mathrm{bp}$ insertion in the promoter region. This observation, however, was not replicated in a population from the northern UK [55]. This particular polymorphism was not interrogated in the

Table 4: Haplotype association with risks of conotruncal heart defects

\begin{tabular}{lcc}
\hline Haplotype & Frequency & Odds Ratios $(\mathbf{9 5 \%} \mathbf{C l})$ \\
\hline Block 19 (MTR) & & REF \\
AATCTTTCCTAGAGGGCTTGG & 0.354 & $1.1(0.7-1.5)$ \\
GTGGCCCTGGGAAGAAGAGAT & 0.272 & $1.2(0.8-1.8)$ \\
GTGGCCCTCTAGGTGACTTGG & 0.189 & $1.5(0.8-3.0)$ \\
GTGGCCTTCTAGATGACTTGT & 0.048 & $1.0(0.5-2.1)$ \\
GTGGCCCTCGAAAGGAGTTGT & 0.035 & $0.9(0.3-2.2)$ \\
GTGGCCCTGGGGAGAAGAGAT & 0.021 & $10.7(1.4-84.8)$ \\
GATCTTTCCTAGAGGGCTTGG & 0.013 & \\
\hline
\end{tabular}

Block 19 included rs4659724, rs955516, rs4077829, rs 12060570, rs 1806505, rs6668344, rs3754255, rs 10925252, rs3768139, rs3768।42, rs 1770449, rs7367859, rs 1805087, rs2275565, rs 1266164, rs2229276, rs 10802569, rs4659743, rs382057I, rs 1050993, and rs6676866. 
current study. Three of the five TYMS SNPs (rs284179, rs1001761, and rs502396) investigated here showed elevated risks for spina bifida for both heterozygote or homozygote individuals. This finding and the corresponding haplotype finding (Table 3) will be important to explore in future studies.

The strengths of this study were: 1) it investigated the potential effects of a large number of folate pathway SNPs, as well as investigated haplotype associations; 2) it had population-based ascertainment of two case phenotypes and controls; and 3) it included cases and controls born before the US food supply was fortified with folic acid, thus we would expect a sizable proportion of cases to have been folate-responsive.

Conversely, our study was limited in its effect estimation owing to small sample sizes for some comparisons. For example, our study had $80 \%$ power to detect risks of 2.5 or more associated with genotypes that were observed in at least $4 \%$ of controls. Another potential limitation is the lack of information on maternal folate status. Our working hypothesis is that transient elevation in maternal serum folate from supplementation or dietary intake could prevent birth defects by overcoming metabolic inefficiencies or transport-related issues. Absence of information on low folate status would make it more difficult to find putative genotypes. It is also possible that the protective effect of folic acid relates to correction of a maternal metabolic defect, rather than the fetus. Our study was limited to infant genotype information. Thus, we were unable to investigate the potential effects of maternal genotype. As with any study that seeks to explore associations with a large number of genotypes, findings are subject to chance owing to multiple comparisons. As noted above, we conducted 472 analytic comparisons and thus expected more "statistically significant" findings to arise by chance alone. Further, our findings may have been influenced by uncontrolled confounding by population stratification undetectable in analyses stratified or adjusted by race/ethnicity $[60,61]$. Lastly, the selected SNPs represent only a fraction of the potential variation of the studied genes. Thus, full gene coverage was not achieved even though a large number of SNPs was studied.

\section{Conclusion}

Despite compelling evidence that folate intake by women in early pregnancy substantially reduces risks of selected birth defects, the underlying mechanisms have not been elucidated. Our study attempted to determine genetic mechanisms responsible for folic acid's preventive effects. Our observations do not implicate a particular folate transport or metabolism gene to be strongly associated with risks for spina bifida or conotruncal defects. Although we explored a sizable number of polymorphic areas in these genes, we clearly did not capture all the genetic variation. Thus, these genes may continue to be candidates for further inquiry. Alternatively, the preventive role of folate may be via other biological mechanisms such as methylation of nonfolate-related genes that participate in the closure of the neural tube or the development of the heart.

\section{Competing interests}

The authors declare that they have no competing interests.

\section{Authors' contributions}

GMS conceived of the study and participated in the statistical analysis. WL conducted the molecular genetic studies. HZ conducted the molecular genetic studies and participated in the statistical analysis. WY conducted the statistical analysis. FBSB conducted the statistical analysis. SLC participated in the statistical analysis. LFB designed and participated in the statistical analysis. EJL conceived of the study and participated in the statistical analysis. RHF conceived of the study and directed the laboratory molecular genetic studies. All authors read and approved the final manuscript.

\section{Additional material}

\section{Additional file 1}

Appendix. Risks of spina bifida and conotruncal heart defects among California infants associated with 118 SNPs in 14 genes involved in folate metabolism or transport relative to nonmalformed population-based controls.

Click here for file

[http://www.biomedcentral.com/content/supplementary/14712350-10-49-S1.doc]

\section{Acknowledgements}

This research was supported by funds from the Centers for Disease Control and Prevention, Center of Excellence Award U50/CCU91324I, by $\mathrm{NIH/NHLBI} \mathrm{ROI} \mathrm{HL085859,} \mathrm{and} \mathrm{by} \mathrm{NIH/NINDS} \mathrm{ROI} \mathrm{NS050249.} \mathrm{We} \mathrm{thank}$ the California Department of Public Health Maternal Child and Adolescent Health Division for providing data for these analyses. The findings and conclusions in this report are those of the authors and do not necessarily represent the views of the California Department of Public Health.

\section{References}

I. Prevention of neural tube defects: results of the Medical Research Council vitamin study. MRC Vitamin Study Research Group. Lancet 199|, 338(8760):131-7.

2. Czeizel $A E$, Dudás I: Prevention of the first occurrence of neural-tube defects by periconceptional vitamin supplementation. N Engl J Med 1992, 327(26): 1832-5.

3. Shaw GM, Lammer EJ, Wasserman CR, O'Malley CD, Tolarova MM: Risks of orofacial clefts in children born to women using multivitamins containing folic acid periconceptionally. Lancet 1995, 346:393-6.

4. Shaw GM, O'Malley CD, Wasserman CR, Tolarova MM, Lammer EJ: Maternal periconceptional use of multivitamins and reduced 
risk for conotruncal heart defects and limb deficiencies among offspring. Am J Med Genet 1995, 59:536-45.

5. Botto LD, Mulinare J, Erickson JD: Occurrence of congenital heart defects in relation to maternal multivitamin use. $\mathrm{Am} J$ Epidemiol 2000, I 5 I(9):878-84.

6. Czeizel $A E$, Tüth M, Rockenbauer M: Population-based case-control study of folic acid supplementation during pregnancy. Teratology 1996, 53(6):345-5I.

7. Werler MM, Hayes C, Louik C, Shapiro S, Mitchell AA: Multivitamin supplementation and risk of birth defects. Am J Epidemiol 1999 , I 50(7):675-82.

8. Loffredo LC, Souza JM, Freitas JA, Mossey PA: Oral clefts and vitamin supplementation. Cleft Palate Craniofac J 200I, 38(I):76-83.

9. Itikala PR, Watkins ML, Mulinare J, Moore CA, Liu Y: Maternal multivitamin use and orofacial clefts in offspring. Teratology 200 I, 63(2):79-86

10. Czeizel AE: Reduction of urinary tract and cardiovascular defects by periconceptional multivitamin supplementation. Am J Med Genet 1996, 62(2): 179-83.

II. Czeizel AE, Dobó M, Vargha P: Hungarian cohort-controlled trial of periconceptional multivitamin supplementation shows a reduction in certain congenital abnormalities. Birth Defects Res A Clin Mol Teratol 2004, 70(I I):853-6I.

12. Put NM van der, Steegers-Theunissen RP, Frosst P, Trijbels FJ, Eskes TK, Heuvel LP van den, Mariman EC, den Heyer M, Rozen R, Blom $\mathrm{HJ}$ : Mutated methylenetetrahydrofolate reductase as a risk factor for spina bifida. Lancet 1995, 346(8982): I070-I.

13. Put NM van der, Heuvel LP van den, Steegers-Theunissen RP, Trijbels FJ, Eskes TK, Mariman EC, den Heyer M, Blom HJ: Decreased methylene tetrahydrofolate reductase activity due to the 677C-->T mutation in families with spina bifida offspring. J Mol Med 1996, 74(I I):69I-4.

14. Kirke PN, Mills JL, Whitehead AS, Molloy A, Scott JM: Methylenetetrahydrofolate reductase mutation and neural tube defects. Lancet 1996, 348(9033): 1037-8.

15. Shaw GM, Rozen R, Finnell RH, Wasserman CR, Lammer EJ: Maternal vitamin use, genetic variations of infant methylene tetrahydrofolate reductase and risk for spina bifida. $\mathrm{Am} J$ Epidemiol 1998, I48(I):30-7.

16. Posey DL, Khoury MJ, Mulinare J, Admas MJ Jr, Ou CY: Is mutated MTHFR a risk factor for neural tube defects? Lancet 1996, 347(9002):686-7.

17. Botto LD, Yang Q: 5, 10-Methylenetetrahydrofolate reductase gene variants and congenital anomalies: a HuGE review. Am J Epidemiol 2000, I5 I (9):862-77.

18. Junker R, Kotthoff S, Vielhaber H, Halimeh S, Kosch A, Koch HG, Kassenböhmer R, Heineking B, Nowak-Göttl U: Infant methylenetetrahydrofolate reductase 677TT genotype is a risk factor for congenital heart disease. Cardiovas Res 200I, 5 I (2):25I-4.

19. Wenstrom KD, Johanning GL, Johnston KE, DuBard M: Association of the C677T methylenetetrahydrofolate reductase mutation and elevated homocysteine levels with congenital cardiac malformations. Am J Obstet Gynecol 200I, 184(5):806-I7.

20. Croen LA, Shaw GM, Jensvold NJ, Harris JA: Birth defects monitoring in California: a resource for epidemiological research. Paediatr Perinat Epidemiol I99|, 5(4):423-7.

2I. Schulman J, Hahn JA: Quality control of birth defects registry data: a case study. Publ Health Rep 1993, 108(I):91-8.

22. Lovmar L, Syvänen AC: Multiple displacement amplification to create a long-lasting source of DNA for genetic studies. Hum Mutat 2006, 27(I):603-14.

23. Holbrook JF, Stabley D, Sol-Church K: Exploring whole genome amplification as a DNA recovery tool for molecular genetic studies. J Biomol Tech 2005, I 6(2): I25-33.

24. Bergen AW, Qi Y, Haque KA, Welch RA, Chanock SJ: Effects of DNA mass on multiple displacement whole genome amplification and genotyping performance. BMC Biotechnol 2005, 5:24.

25. Fredicksen A, Meyer K, Ueland PM, Vollset SE, Grotmol T, Schneede $\mathrm{J}$ : Large-scale population-based metabolic phenotyping of thirteen genetic polymorphisms related to one-carbon metabolism. Human Mut 2007, 28(9):856-65.

26. Piedrahita JA, Oetma B, Bennett GD, van Waes J, Kamen BA, Richardson J, Lacey SW, Anderson RG, Finnell RH: Mice lacking the folic-acid binding protein Folbpl are defective in early embryonic development. Nat Genet 1999, 23(2):228-32.
27. Linden IJ van der, Afman LA, Heil SG, Blom HJ: Genetic variation in genes of folate metabolism and neural-tube defect risk. Proc Nutr Soc 2006, 65(2):204-I5.

28. Boyles AL, Billups AV, Deak KL, Siegel DG, Mehltretter L, Slifer SH, Bassuk AG, Kessler JA, Reed MC, Nijhout HF, George TM, Enterline DS, Gilbert JR, Speer MC, NTD Collaborative Group: Neural tube defects and folate pathway genes: family-based association tests of gene-gene and gene-environment interactions. Environ Health Perspect 2006, I I 4( I 0): I547-52.

29. Hobbs CA, James SJ, Parsian A, Krakowiak PA, Jerrigan S, Greenhaw JJ, Lu Y, Cleves MA: Congenital heart defects and genetic variants in the methylenetetrahydrofolate reductase gene. J Med Genet 2006, 43(2): 162-6.

30. Shaw GM, lovannisci DM, Yang W, Finnell RH, Carmichael SL, Cheng $S$, Lammer EJ: Risks of human conotruncal heart defects associated with 32 single nucleotide polymorphisms of selected cardiovascular disease-related genes. Am J Med Genet A 2005, I38(I):2I-6.

31. Storti S, Vittorini S, Lascone MR, Sacchelli M, Collavoli A, Ripoli A, Cocchi G, Biagini A, Clerico A: Association between 5, 10-methylenetetrahydrofolate reductase C677T and AI298C polymorphisms and conotruncal heart defects. Clin Chem Lab Med 2003, 4 I (3):276-80.

32. Deng L, Elmore CL, Lawrance AK, Matthews RG, Rozen R: Methionine synthase reductase deficiency results in adverse reproductive outcomes and congenital heart defects in mice. Mol Genet Metab 2008, 94(3):336-42.

33. Zhu H, Wicker NJ, Shaw GM, Lammer EJ, Hendricks K, Suarez L, Canfield M, Finnell RH: Homocysteine remethylation enzyme polymorphisms and increased risks for neural tube defects. Mol Genet Metab 2003, 78(3):2I6-2I.

34. Doolin MT, Barbaux S, McDonnell M, Hoess K, Whitehead AS, Mitchell LE: Maternal genetic effects, exerted by genes involved in homocysteine remethylation, influence the risk of spina bifida. Am J Hum Genet 2002, 7 I (5): I 222-6.

35. Guçant-Rodriguez RM, Rendeli C, Namour B, Venuti L, Romano A, Anello G, Bosco P, Debard R, Gçrard P, Viola M, Salvaggio E, Guçant $\mathrm{JL}$ : Transcobalamin and methionine synthase reductase mutated polymorphisms aggravate the risk of neural tube defects in humans. Neurosci Lett 2003, 344(3): 189-92.

36. De Marco P, Calevo MG, Moroni A, Arata L, Merello E, Finnell RH, Zhu H, Andreussi L, Cama A, Capra V: Study of MTHFR and MS polymorphisms as risk factors for NTD in the Italian population. J Hum Genet 2002, 47(6):319-24.

37. O'Leary VB, Mills JL, Pangilinan F, Kirke PN, Cox C, Conley M, Weiler A, Peng K, Shane B, Scott JM, Parle-McDermott A, Molly AM, Brody LC, Members of the Birth Defects Research Group: Analysis of methionine synthase reductase polymorphisms for neural tube defects risk association. Mol Genet Metab 2005, 85(3):220-7.

38. Banerjee R, Zou CG: Redox regulation and reaction mechanism of human cystathionine-B-synthase: a PLP-dependent hemesensor protein. Arch Biochem Biophys 2005, 433(I): I 44-56.

39. Wilson A, Platt R, Wu Q, Leclerc D, Christensen B, Yang $H$, Gravel RA, Rozen R: A common variant in methionine synthase reductase combined with low cobalamin (vitamin BI2) increases risk for spina bifida. Mol Genet Metab 1999, 67(4):317-23.

40. Candito M, Rivet R, Herbeth B, Boisson C, Rudigoz RC, Luton D, Journel H, Oury JF, Rouv F, Saura R, Vernhet I, Gaucherand P, Muller F, Guidicelli B, Heckenroth H, Poulain P, Blayau M, Francannet C, Roszy KL, Brustiç C, Staccini P, Gçrard P, Fillion-Emery N, GuçantRodriguez RM, Van Obberghen E, Guçant JL: Nutritional and genetic determinants of vitamin $B$ and homocysteine metabolisms in neural tube defects: a multicenter case-control study. Am J Med Genet Part A 2008, I46A(9): I I28-33.

41. Relton CL, Wilding CS, Laffling AJ, Jonas PA, Burgess T, Binks K, Tawn EJ, Burn J: Low erythrocyte folate status and polymorphic variation in folate-related genes are associated with risk of neural tube defect pregnancy. Mol Genet Metabol 2004, 8I(4):273-8I.

42. van Beynum IM, Kouwenberg M, Kapusta L, den Heijer M, Linden II van der, Daniels O, Blom H]: MTRR 66A>G polymorphism in relation to congenital heart defects. Clin Chem Lab Med 2006, 44(II):1317-23. 
43. Verkleij-Hagoort AC, van Driel LM, Lindemans J, Isaacs A, Steegers EA, Helbing WA, Uitterlinden AG, Steegers-Theunissen RP: Genetic lifestyle factors related to the periconception vitamin B 12 status and congenital heart defects: a Dutch case-control study. Mol Genet Metab 2008, 94(I): I I2-9.

44. Parle-McDermott A, Kirke PN, Mills JL, Molloy AM, Cox C, O'Leary VB, Pangilinan F, Conley M, Cleary L, Brody LC, Scott JM: Confirmation of the $\mathrm{R} 653 \mathrm{Q}$ polymorphism of the trifunctional CI-synthase enzyme as a maternal risk for neural tube defects in the Irish population. Eur J Hum Genet 2006, I4(6):768-72.

45. De Marco P, Merello E, Calevo MG, Mascelli S, Raso A, Cama A, Capra V: Evaluation of a methylenetetrahydrofolate-dehydrogenase 1958>A polymorphism for neural tube defect risk. J Hum Genet 2006, 5 I (2):98-103.

46. Johnson WG, Stenroos ES, Spychala JR, Chatkupt S, Ming SX, Buyske $S$ : New 19 bp deletion polymorphism in Intron-I of dihydrofolate reductase (DHFR): a risk factor for spina bifida acting in mothers during pregnancy? Am J Med Genet A 2004, I 24A(4):339-45.

47. Parle-McDermott A, Pangilinan F, Mills JL, Kirke PN, Gibney ER, Troendle J, O'Leary VB, Molloy AM, Conley M, Scott JM, Brody LC: The 19-bp deletion polymorphism in Intron-I of dihyrofolate reductase (DHFR) may decrease rather than increase risk for spina bifida in the Irish population. Am J Med Genet A 2007, I 43A(I I): I I 74-80.

48. Linden IJ van der, Nguyen U, Heil SG, Franke B, Vloet S, Gellekink H, den Heijer M, Blom HJ: Variation and expression of dihydrofolate reductase (DHFR) in relation to spina bifida. Mol Genet Met 2007, 9 I(I):98-103.

49. Shaw GM, Lammer EJ, Zhu H, Baker MW, Neri E, Finnell RH: Maternal periconceptional vitamin use, genetic variation of infant reduced folate carrier (A80G), and risk of spina bifida. $\mathrm{Am} J$ Med Genet 2002, I08(I): I-6.

50. Shaw GM, Zhu H, Lamer E], Yang W, Finnell RH: Genetic variation of infant reduced folate carrier (A80G) and risk of orofacial and conotruncal heart defects. Am J Epidemiol 2003, I 58(8):747-52.

5I. Pei L, Zhu H, Ren A, Li Z, Hao L, Finnell RH, Li Z: Reduced folate carrier gene is a risk factor for neural tube defects in a Chinese population. Birth Defects Res A Clin Mol Teratol 2005, 73(6):430-3.

52. Pei L, Zhu H, Zhu J, Ren A, Finnell RH, Li Z: Genetic variation of infant reduced folate carrier (A80G) and risk of orofacial defects and congenital heart defects in China. Ann Epidemiol 2006, 16(5):352-6.

53. De Marco P, Calevo MG, Moroni A, Merello E, Raso A, Finnell RH, Zhu H, Andreussi L, Cama A, Capra V: Reduced folate carrier polymorphism (80A-->G) and neural tube defects. Eur J Hum Genet 2003, I I(3):245-52.

54. Trinh BN, Ong CN, Coetzee GA, Yu MC, Laird PW: Thymidylate synthase: a novel genetic determinant of plasma homocysteine and folate levels. Hum Genet 2002, I I I (3):299-302.

55. Wilding CS, Relton CL, Sutton MJ, Jonas PA, Lynch SA, Tawn EJ, Burn $\mathrm{J}$ : Thymidylate synthase repeat polymorphisms and risk of neural tube defects in a population from the Northern United Kingdom. Birth Def Res A Clin Mol Teratol 2004, 70(7):483-5

56. Volcik KA, Shaw GM, Zhu H, Lammer EJ, Laurent C, Finnell RH: Associations between polymorphisms within the thymidylate synthase gene and spina bifida. Birth Defects Res A Clin Mol Teratol 2003, 67(II):924-8.

57. Liu J, Schmitz JC, Lin X, Tai N, Yan W, Farrell M, Bailly M, Chen T, Chu $E$ : Thymidylate synthase as a translational regulator of cellular gene expression. Biochim Biophys Acta 2002, I 587(23): $174-82$.

58. Kawate $\mathrm{H}$, Landis DM, Loeb LA: Distribution of mutations in human thymidylate synthase yielding resistance to 5 -fluorodeoxyuridine. J Biol Chem 2002, 277(39):36304-II.

59. Ulrich CM, Bigler J, Bostick R, Fosdick L, Potter JD: Thymidylate synthase promoter polymorphism, interaction with folate intake, and risk of colorectal adenomas. Cancer Res 2002, 62(12):336I-4.

60. Thomas DC, Witte JS: Point: population stratification: a problem for case-control studies of candidate-gene associations? Cancer Epidemiol Biomarkers Prev 2002, I I (6):505- I2.
61. Wacholder S, Rothman N, Caporaso N: Counterpoint: bias from population stratification is not a major threat to the validity of conclusions from epidemiological studies of common polymorphisms and cancer. Cancer Epidemiol Biomarkers Prev 2002. II (6):513-20.

\section{Pre-publication history}

The pre-publication history for this paper can be accessed here:

\section{http://www.biomedcentral.com/1471-2350/10/49/pre} pub
Publish with Bio Med Central and every scientist can read your work free of charge

"BioMed Central will be the most significant development for disseminating the results of biomedical research in our lifetime. "

Sir Paul Nurse, Cancer Research UK

Your research papers will be:

- available free of charge to the entire biomedical community

- peer reviewed and published immediately upon acceptance

- cited in PubMed and archived on PubMed Central

- yours - you keep the copyright 